\title{
When my avatar's movements make me feel I am moving: From natural-like stimuli to fully artificial ones in virtual reality
}

\section{Quand les mouvements de mon avatar me donnent l'impression que je bouge : des stimuli naturels à des stimuli entièrement artificiels en réalité virtuelle}

\author{
Marion Giroux, Julien Barra, Christian Graff, and Michel Guerraz \\ Univ. Grenoble Alpes, Univ. Savoie Mont Blanc, CNRS, LPNC, 38000 Grenoble, France
}

\begin{abstract}
In virtual reality, users do not receive any visual information coming from their own body. Thus, avatars are often used, and they can be embodied which alters the body representation. We suggested that the perception of one's own movements (i.e., kinaesthesia) can be altered as well. We investigated whether visual cues coming from an avatar can be used for kinaesthesia and to what extent such cues can deviate from natural ones. We used a paradigm in which the participant's left forearm was moved passively, correlated with the movement of both forearms of the avatar. Such visuo-proprioceptive combination induces kinaesthetic illusions in the participant's right forearm. The impact of the morphological similarity (semantic congruency) and of the visual perspective of the avatar (spatial congruency) was investigated. Results have indicated that avatar's movements are processed as one's own movements. Morphological similarity and first-person perspective were not necessary, but they reinforced the illusions. Thus, visual motion cues can strongly deviate from natural ones in morphology and perspective and still contribute to kinaesthesia.
\end{abstract}

\begin{abstract}
Résumé. En réalité virtuelle, les utilisateurs ne reçoivent aucune information visuelle de leur propre corps. Des avatars sont souvent utilisés pour combler ce manque. Ces avatars peuvent être incarnés et modifier la représentation que l'utilisateur se fait de son corps. Ainsi, si l'avatar peut être assimilé au corps de l'utilisateur, les mouvements de cet avatar pourraient également être assimilés à des mouvements du corps propre et ainsi affecter sa perception du mouvement. Nous avons utilisé un paradigme dans lequel le bras gauche du participant était déplacé passivement, en corrélation avec le mouvement des deux bras de l'avatar. Cette combinaison visuo-proprioceptive induisait des illusions de mouvement dans le bras droit du participant témoignant de l'impact de l'avatar sur la kinesthésie. Les importances respectives de la similarité morphologique et d'une perspective visuelle à la première personne de l'avatar ont également été étudiées et elles s'avèrent non nécessaires, même si elles renforcent les illusions. Ainsi, les informations visuelles de mouvement provenant de l'avatar peuvent grandement différer des informations naturelles liées au corps propre et néanmoins contribuer à la kinesthésie.
\end{abstract}




\section{Avatar embodiment in virtual reality}

In virtual reality, a virtual environment is generated by a computer and displayed to the users through screens or Head Mounted Displays (HMD). The latter devices capture the movements of the users' head which enables a natural-like exploration of the virtual environment in real time. When wearing an HMD, the only visual information received by users are artificial ones created by the computer. Thus, users do not receive any visual information coming from their own body which could lead to the feeling of not having a body, i.e., to be disembodied. Numerous set-ups in virtual reality choose to represent the user's body in the virtual environment by an avatar to limit this possibly uncomfortable feeling since such avatar can be "embodied". The feeling of embodiment has been defined by different authors in more or less specific situations. De Vignemont approached the question of embodiment in a general way and suggested that an "object $\mathrm{E}$ is embodied if some properties of $\mathrm{E}$ are processed in the same way as the properties of one's body" (de Vignemont, 2011). Kilteni and colleagues (2012), for their part, considered that a feeling of embodiment towards a body $\mathrm{B}$ occurs if and only if, at least one of the following three feelings is felt with at least minimal intensity:

- Feeling of being localised in the body B (Self-location);

- Feeling of being the agent of the movements of the body B (Agency);

- Feeling that the body B is one's own (Ownership).

These three sub-components would be considered and combined into a general feeling of embodiment toward the body B. The resulting feeling could be represented on a continuous scale indicating various possible degrees ranging from an "absence of feeling of embodiment" to a "maximal intensity of this feeling", the latter being reached, a priori, only for the real body (Kilteni, et al., 2012). Both definitions apply to the real physical body as well as to an avatar, a prosthesis, a tool or fake body parts. In a more specific way, Gonzalez-Franco \& Peck (2018) defined the embodiment of an avatar as the "illusory feeling that the avatar has replaced one's own body at a physical and/or functional level in the virtual environment" which includes the sub-components described by Kilteni et al., (2012). These sub-components have been highlighted by several studies dealing with embodiment illusions (i.e., illusory feeling to embody a fake or virtual body). For example, Longo and colleagues used a principal component analysis to segregate ownership, agency and self-location during the Rubber Hand Illusion (Longo et al., 2008). This illusion occurs when a fake hand and the real hand of the participant are stroked synchronously while the latter is hidden from view. Such visuo-tactile stimulation leads to a feeling of embodiment toward the rubber hand that is notably objectivised by a real hand-withdrawal reflex when the rubber hand is struck with a mallet (Botvinick et Cohen, 1998).

In a typical way, embodiment is felt toward one's own body but it can be affected in some pathologies (i.e., some body parts or the whole body felt like they are no longer part of oneself) (Barra et al., 2020 for a review) or, as explained above, it can stretch to external objects like a rubber hand or an avatar. Many studies reported the occurrence of a feeling of embodiment toward a first-person perspective avatar (e.g., Normand et al., 2011; Slater et al., 2010). For example, in the experiment conducted by Slater and colleagues (2010) with adult participants, a feeling of ownership towards a little girl avatar seen at the first-person perspective, was observed. The participants could also see their avatar being touched on the shoulder and feel the corresponding touch on their own shoulder. Such synchronous visuo-tactile stimulation also enhanced the feeling of ownership towards the avatar (Slater et al., 2010). Likewise, a study from Lugrin et al. (2015) reported high level of illusion of virtual body ownership towards first-person avatars with different levels of anthropomorphism. In this experiment, the avatar replicated in real time every movements of the participant's body (Lugrin et al., 2015). Our own studies also showed the embodiment of first-person perspective avatars whose forearms could be moved in real time by the participant (Giroux et al., 2018, 2019).

It is important to note that in the experiments evoked above, synchronous multisensory or sensorimotor stimulations (visuo-tactile: Slater et al., 2010; visuomotor: Lugrin et al., 2015 and Giroux et al., 2018; 2019) were at the root of the avatar's embodiment or, at the very least, increased such feeling. Moreover, the first-person perspective itself can be enough to induce a feeling of embodiment (when the avatar is sufficiently realistic) since there is still a congruent visuo-proprioceptive stimulation. Indeed, at the first-person perspective, there is, with some exceptions, a congruency between visual information coming from the avatar's body and proprioceptive information about the real body posture (Maselli et Slater, 2013). Furthermore, most of head-mounted displays, used nowadays in virtual reality set-ups, can track in real-time users' head movements. Such feature enables the participants to explore the virtual environment by naturally moving their head, but it can also be used to animate the avatar's head in congruence with the users' head, inducing a visuo-motor congruency when the users see their avatar in a mirror (e.g., Slater et al., 2010). Thus, the embodiment of first-person perspective avatars appears to be relatively straightforward provided that some multisensory or sensorimotor congruencies between the avatar and the real body are supplied. Moreover, the avatar does not have to be a realistic representation of the human body to be embodied, especially when it is induced by visuo-motor combination. Indeed, in the literature, there are many examples of embodiment of non-humanoid objects ranging from a cat's paw (Zhang et Hommel, 2016) to dots placed at the fingertips' locations (Schwind et al., 2018), including a wooden block (Lin et Jörg, 2016) or even a 2D square (Ma et Hommel, 2015). 


\section{The impact of avatar embodiment on mental body representations}

The embodiment of an avatar has many impacts on action and cognition, including the proteus effect. In the proteus effect, identity cues derived from the visual characteristics of the avatar alter the user's behaviours and attitudes (Yee \& Bailenson, 2007). For instance, Beaudoin et al. (2020) showed that embodiment of an avatar resembling an elderly adult by young adult participants can change mentally-represented physical activity in a motor imagery task. In addition, the more negative the participant's beliefs about motor activity in the elderly, the greater the impact of the avatar on motor imagery performance. Therefore, the knowledge about the characteristics of an embodied avatar can modify the participant's level of mentally represented physical activity. In the same vein, the attractiveness of the embodied avatar impacts the degree of intimacy expressed by the participant's behaviour towards confederates (Yee et Bailenson, 2007) and embodiment of a dark-skinned avatar leads reduced implicit racial bias in white-skinned people (Peck et al., 2013). More interestingly for our purpose, avatar embodiment can also impact our mental body representations.

The fact that mental body representations exist is commonly accepted in the literature, although there are still many debates about the identity, denomination, and number of these representations. A relative consensus seems to admit the existence of an opposition between the body schema and the body image, which are multimodal representations of the body used for different purposes. These terms are widely used in the literature, not always with the same meaning. The body schema is mainly described as a global and modular representation of our body, its shape, its position and its movements that allows us to plan and execute our actions. This representation of the body is considered to be essential to enable us to act and is even considered as a representation of the body for action or motor control (Gallagher, 2005; Head et Holmes, 1911). Indeed, in order to perform an action, such as grasping a piece of cake, we need to know where the cake is located, but also where our arm and hand are in space, in what posture is our hand and what are the shape and size of our arm to determine whether or not we can reach the cake (e.g., too far away, in a box that is too small). All this information is necessary to determine what gestures we need to make to carry out our action and it would be the body schema that gather all this information received from multiple sensory receptors. In addition, the body schema would be constantly updated to consider changes in position but also changes in shape (e.g., weight gain or loss, surgery, amputation) and size (e.g., growth) of the different segments of our body. It would therefore be a real-time ("Online") representation, constructed at each moment by sensory information (Carruthers, 2009).

The question of the body image achieves less consensus than the body schema. Indeed, the unity of body image is greatly questioned because it is often thought of as "everything that is not the body schema" (de Vignemont, 2010). It is considered as a conscious system of perceptions, attitudes and beliefs about one's own body (Gallagher, 2005) and would thus group together perceptual, conceptual and emotional representations of the body (i.e., body percept, body concept and body affect). When the body schema is exclusively a real-time representation, the body image would be composed of both real-time and offline representations. It would therefore include elements constructed in real time using sensory information (e.g., the vision of a bruise on a part of the body) but also more durable elements, which would not be updated easily nor quickly, on what the body usually looks like (e.g., the fact that one has two hands). Body image would not be involved in action in contrast to the body schema.

Therefore, the body schema as well as the body image rely on multisensory information and many studies demonstrated the implication of different sensory signals on body representations, such as visual, tactile or proprioceptive signals (Röder et al., 2013), but also vestibular (Lopez et al., 2012) or auditive (Tajadura-Jiménez et al., 2015) ones. However, it is often not easy to determine which of the body image or body schema is affected by such stimulations mostly since they are probably both. Another way to alter these mental body representations is through embodiment or ownership illusions, which consist in the illusory feeling of embodiment toward external body or body parts. As previously explained, it is possible to have a feeling of embodiment toward virtual but also physical external objects provided that multisensory stimulations (e.g., visuo-tactile, visuo-motor) initiate it. When such an embodied external object differs on some characteristics from the real body, the mental body representations are modified. For example, when looking at a life-size picture of a face placed in front of their face and being touched simultaneously and at the same location (i.e., visuo-tactile stimulation) like if it were their face reflected by a mirror, participants see the mental representations of their face altered. The embodiment of the face seen is called the enfacement illusion (Sforza et al., 2010) and has consequences on the other/self discrimination. Indeed, participants attribute more facial features from the other face to their own in other/self discrimination and recognition tasks compared to a condition with incongruent visuo-tactile stimulation (i.e., without illusory embodiment) (Sforza et al., 2010; Tsakiris, 2008). In the same idea, the "very long arm illusion" used visuo-motor stimulation to induce an illusory feeling of embodiment toward a virtual arm, colocalised with the real arm of the participant. This virtual arm can slowly lengthen until it reaches twice, three or four times its original length. When the hand at the end of this very long virtual arm is threatened (by a virtual saw), participants have withdrawal hand movements. Thus, the mental representations of the arm have changed, lengthening with the virtual arm the participants see (Kilteni, Normand, et al., 2012). Similar results are found when, in virtual reality, participants embody a first-person perspective avatar with a larger belly than theirs. Indeed, after such embodiment, participants overestimate their own body size. The representations of their own body are altered by the embodiment of the large-belly avatar, including characteristics from the latter (Normand et al., 2011).

To summarize, multisensory or sensorimotor stimulations can generate a feeling of embodiment towards an external body or body parts either virtual or physical. Such external objects can be processed as one's own body as they impact mental body representations and, more precisely, the representation of the body shape and size. Some characteristics 
from the embodied object are attributed to one's own body. Thus, embodiment of external objects has consequences on the perception of one's own body but also on the perception of one's own movements as we will see below.

\section{Kinaesthesia}

Before exposing the consequences of embodiment on kinaesthesia, we must define and describe what we mean by kinaesthesia. Kinaesthesia consists in our ability to consciously perceive movements of our body parts in space. We have a conscious access to a kinaesthetic percept, that is a feeling of movement, and this percept results from the integration of different signals both peripheral (sensory) and central (motor). No specific sensory signal is devoted to kinaesthesia but all body sensory signals available contribute to the construction of the sense of movement. The implication of visual, proprioceptive, tactile, and auditive signals in kinaesthesia has been largely demonstrated through the existence of movement's illusion induced by specific sensory manipulations.

An illusory feeling of movement of one's own whole body can be induced by the vision of a moving visual flow in a static observer (Brandt et al., 1972; Guerraz et Bronstein, 2008; Harquel et al., 2020). Such visual illusion, called vection, can be experienced when we are in a train at a station stop and the train next to us starts moving. For a moment, it feels like our train is moving. In this situation, the visual flow we receive indicates a movement of almost our entire environment, which generally corresponds to a movement of our body in space. If, as we are experiencing this feeling of vection, we fix our gaze on the station platform (which is static) for example, it will immediately disappear, the ambiguity between movement of an external object and movement of our own body will be removed. Experimentally, there are different ways of generating these phenomena by scrolling or rotating a visual pattern (e.g., black dots on a white background) to create the illusion of linear displacement, called linear vection, in different directions in space (front-back; up-down; right-left), or rotation, called circular vection (Brandt et al., 1972). Nowadays, it is also possible to induce these vection phenomena through virtual reality. Indeed, it is possible to expose the participant to a more or less complex visual flow in order to induce feelings of vection (Gallagher et al., 2019; Kuiper et al., 2019). It is also possible to induce such a perception of movement of parts of the body on the same principle as that used for whole body. Indeed, the rotation of a visual pattern under a participant's hand (or arm) leads to the sensation of a rotating movement of the wrist (or elbow) in the opposite direction (Blanchard et al., 2013; Chancel et al., 2016; Tardy-Gervet et al., 1984).

Vections can also be induced by auditory signal manipulations. Indeed, when blindfolded, the exposure to moving sounds or, more effectively, to a moving sound field leads to the feeling of being in motion, either in rotation (i.e., circular vection) when the sound field rotates or in translation (i.e., linear vection) when it moves linearly (Väljamäe, 2009). In addition, sounds related to our actions and, even more effectively, the sonification of movements - which transforms movement characteristics (e.g., speed, spatial orientation) into an acoustic signal - can be used to improve motor performance (Effenberg, 2004), motor learning (Effenberg et al., 2015) or motor rehabilitation (Bevilacqua et al., 2018; Maulucci et Eckhouse, 2001). Thus, these auditory stimuli influence the performance in these multiple motor tasks, suggesting that they may also contribute to limb movement perception, although no studies to our knowledge have directly addressed this issue.

If the existence of illusory feelings of limb movement induced by auditive manipulation remains to be demonstrated, such illusion has been proven to be strongly induced by muscle proprioceptive signal (i.e., the signals coming from the muscles spindles) manipulations. For example, the vibration of the elbow flexor generates the illusion of an extension while the vibration of the elbow extensor generates the illusion of a flexion (Proske et Gandevia, 2012). Furthermore, the simultaneous vibration of two antagonistic muscles at the same frequency does not lead to any illusion of movement (Calvin-Figuière et al., 1999) but degrades or masks proprioceptive signals, resulting in impaired performance in sensorimotor tasks (e.g., wrist angle matching task, force production) (Bock et al., 2007; Brun et Guerraz, 2015). These proprioceptive afferences would be integrated between the hemispheres, at least with respect to the upper limbs, resulting in bimanual coupling. Indeed, the manipulation of proprioceptive information in one arm affects both the motor behaviour of the other arm (Brun et al., 2015; Brun et Guerraz, 2015) and its perceived movements and position (Guerraz et al., 2012; Izumizaki et al., 2010). For example, the speed of the Kohnstamm phenomenon - an involuntary motor response following a sustained and prolonged isometric contraction of the arm muscles - will match the speed of passive movement of the other arm unless the proprioceptive information of the other arm is masked by simultaneous vibration of the antagonistic muscles (Brun et Guerraz, 2015). Thus, ipsi- and contralateral muscle proprioception play an important role in kinaesthesia.

Lastly, manipulations of tactile signal have also been proven to induce body illusions by rotating a platform or applying a specific tactile pattern to the foot sole (Lackner et DiZio, 1984; R. Roll et al., 2002). Similarly, the rotation of a textured disc under a participant's hand induces an illusion of wrist rotation in the opposite direction (Blanchard et al., 2013; Chancel et al., 2016; Kavounoudias et al., 2008) and simple skin stretching lead to illusions of finger movements (Collins et Prochazka, 1996). 


\section{The impact of avatar embodiment on kinaesthesia}

As we just discussed, vision, hearing, muscle proprioception and touch contribute to movement perception, leading to illusions of movement when manipulated independently and artificially (e.g., visual and auditory vections, vibratory illusions). Thus, in the same way body perception results from multisensory integration, perception of one's own movements is achieved by integrating multiple sensory inputs. That's why, just like body perception, kinaesthesia can be influenced by external objects embodiment. Indeed, when a rubber hand is slowly moved, either laterally or in the sagittal axis, illusions of movement in the same direction occurs in the participant's static hidden hand. However, this illusion occurs only when the fake hand was previously embodied, by use of synchronous visuo-tactile stimulation (i.e., the Rubber Hand Illusion) in comparison to asynchronous stimulation (Metral et Guerraz, 2019). This result indicates that visual motion cues coming from the embodied fake hand were used to perceive one's own movement. The same is true when a whole-body avatar is embodied. Indeed, we conducted a study in virtual reality in which a whole-body avatar seen from the first-person perspective had the appearance of a human body (Figure 1, A). Embodiment of this avatar was achieved by asking participants to move their arms into flexion or extension either in phase or antiphase, at a comfortable, self-paced rhythm. In this embodiment phase, the avatar's arms faithfully reproduced the participant's real movements. Such visuo-motor congruency enabled the embodiment into the avatar what is quite implicit in any firstperson video game. Once embodied, it occurred that passive displacement of one of the participant's arms into flexion or extension by a motorized manipulandum, coupled with the displacement of both avatar's arms (Figure 1) was effective in inducing illusions of movement in the participant's static arm. In this virtual replica of the well-known mirror paradigm, the visual motion cues coming from both arms of the avatar were coupled with proprioceptive motion cues originating from the left participant's arm. It is important to note that the illusion of movement in this bimodal stimulation condition was faster and lasted longer than those reported when the left participant's arm was moved but the avatar's forearms hidden from view (unimodal proprioceptive condition) (Giroux et al., 2018). In this experiment, the illusion was assessed by subjective reports on two parameters: speed and duration. Speed was estimated on a scale ranging from 0 (i.e., no illusion) to 10 (i.e., an illusion of movement as fast as the reference motion of the left arm). Duration, expressed in seconds, was based on the participant's estimation of the beginning and the end of the illusion. Hence, visual motion cues coming from the first-person perspective avatar contributed to kinaesthesia since they enhanced the illusion of movement. Furthermore, the bimodal stimulation generated longer and faster illusions than the unimodal visual condition, in which both participant's arm were static while both avatar's forearms were moved. Thus, visual motion cues coming from the avatar were integrated with contralateral proprioceptive motion cues to create a percept of movement more salient than with any unimodal stimulation, in accordance with the probabilistic (or Bayesian) multisensory integration approaches of perception (e.g., Ernst et Banks, 2002).

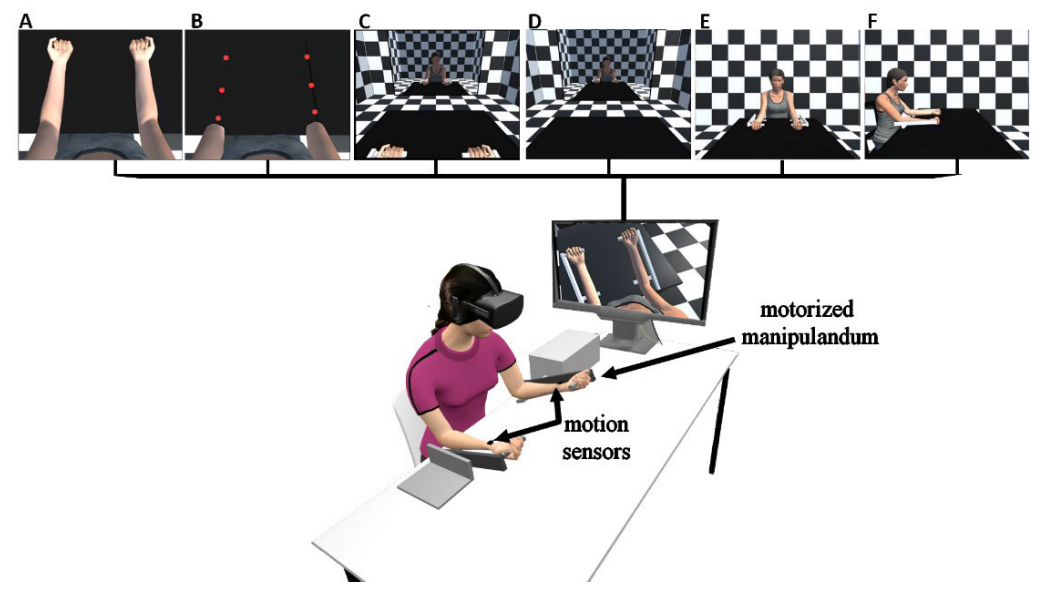

Figure 1. The experimental set-up with the participant wearing a Head-Mounted Display. The virtual reality view is shown on the screen and it differs depending on the experiment and condition. In Giroux et al, 2018, the avatar's arms were represented realistically (A), in Giroux et al., 2019, they were represented by a kind of Point Light Display (B), and in Giroux et al, 2021, the avatar could be seen through a mirror, the $1^{\text {st }}$ person perspective avatar being visible (C) or not (D) or it could be seen at the $3^{\text {rd }}$ person perspective either facing the participant $(\mathrm{E})$ or in profile on one side of the participant (F). Movement of the avatar's forearms were coupled with the passive displacement of the left arm of the participant thanks to the motion sensors.

To go further, the avatar does not have to be represented realistically to be embodied, as seen earlier, and the same applies to its contribution in kinaesthesia. Indeed, in an experiment very similar to the one previously described (Giroux et al., 2018), the avatar's arms were, in some conditions, represented only by three red spheres, inspired by the wellknown Point-Light Displays (Figure 1, B). Embodiment was assessed on nine 7-point Likert scales grading assertions about the participant's feelings. The nine scores were averaged for each participant. The assertions were selected - as 
relevant to our paradigm - from extensive batteries developed by Gonzalez-Franco and Peck (2018). Three components of embodiment feeling were questioned: feeling the avatar's body as one's own (body ownership); feeling control on the avatar's body as on one's own (agency and motor control); feeling the avatar's body located at the same place as one's own (body location). Point-Light Displays are animations made of dots placed on the body key points, such as the joints, and they convey nearly exclusively motion information. Indeed, it is possible to identify many characteristics of the action and the actor when they are seen in motion, but they are incomprehensible when they are static. Thus, the avatar's "Point-light" forearms were not realistic, but they still conveyed visual motion cues. Such avatar representation was enough to generate illusions of movement in the participant right arm. Moreover, a pattern of multisensory integration similar to the one observed with a realistic avatar was found with greater illusion speed and duration in the bimodal visuo-proprioceptive condition than in each unimodal condition (proprioceptive or visual). However, it should be noted that illusions were faster and longer with a realistic avatar than with the Point-Light one (Giroux et al., 2019). Such result indicates that visual motion cues coming from an avatar can still be used in kinaesthesia when there is no morphological correspondence between the avatar and a real human body.

Finally, the avatar does not have to be seen at the first-person perspective, it could be seen through a mirror or at the $3^{\text {rd }}$ person perspective and still contribute to the multimodal percept of movement. Indeed, the same pattern of multisensory integration reported previously was observed when the avatar was no longer seen directly at the first-person perspective but through a mirror facing the participant (Figure 1, D). This means that the kinaesthetic illusions were, once again, faster and longer in the bimodal visuo-proprioceptive condition than in the unimodal proprioceptive condition, even if to a lesser extent than when the first-person perspective avatar's arm were visible (Figure 1, C). Therefore, visual motion cues do not need to be colocalised with the real body to contribute to kinaesthesia since visual information conveyed by a mirror is located inside the mirror. Furthermore, a similar pattern of multisensory integration was found when the avatar was seen at the third-person perspective facing the participant (Figure 1, E). However, it was less obvious when the third-person perspective avatar was seen in profile, on the left or on the right of the participant (Figure 1, F) (Giroux et al., 2021). Thus, visual motion cues do not need to come from a first-person perspective avatar although the contribution of a third-person perspective avatar depends on its location and/or orientation.

Taken together, these results shed light on important features about multisensory integration of artificial visual motion cues in kinaesthesia. Indeed, in order to be integrated, signals from different modalities have to be interpreted as coming from the same source. To this aim, the system focuses on three congruencies, the temporal, the spatial and the semantic congruencies. This means that to be integrated, signals must occur simultaneously, at the same location in space and have to be semantically related (e.g., the sound of an engine and the vision of car). For example, a temporal incongruency between the tactile stimulation and the visual stimulation in the rubber hand illusion fails to induce any feeling of ownership toward the fake hand (Botvinick et Cohen, 1998) as well as a spatial incongruency (e.g., the fake hand being too far from the real one) (Lloyd, 2007; Preston, 2013) or a semantic one (e.g., a wooden block) (Tsakiris et al., 2010). However, these constraints or limits are not as strict as they may appear. Indeed, taking again the example of the Rubber Hand Illusion, some temporal incongruencies can be tolerated by the system. A delay of less than $300 \mathrm{~ms}$ between the visual feedback and the tactile stimulation will not prevent the illusory ownership feeling toward the rubber hand to occur (Shimada et al., 2014). The same applies for the spatial congruency, since the rubber hand have to be close but not exactly at the same place as the real hand to generate the illusion (Botvinick et Cohen, 1998; Preston, 2013 ) and illusions only significantly decline for distance larger than $27.5 \mathrm{~cm}$ (Lloyd, 2007). Also, the rubber hand does not have to be an exact replica of the real hand of the participant since it is a fake hand. Moreover, the illusion occurs even if the rubber hand has not the same skin colour than the participant's (Farmer et al., 2012; Lira et al., 2017). Thus, temporal, spatial and semantic congruencies between sensory signals do not need to be strictly observed to allow multisensory integration. Furthermore, as seen earlier, the impact of incongruencies and more precisely of semantic incongruencies on multisensory integration depends on the modalities involved. Indeed, a wooden block is too far from a realistic hand to generate a feeling of ownership when visuo-tactile stimulation is used (Tsakiris et al., 2010) but the system can overcome such incongruency in condition of visuomotor stimulation (Lin et Jörg, 2016; Ma et Hommel, 2015).

\section{Conclusion}

The results presented above suggest that multisensory integration follows similar rules for both body perception and movement perception. Firstly, an avatar, like a rubber hand, is a fake body. Even if it is a humanoid avatar, it does not look like a real body and, except perhaps in some very recent video games, it cannot be confused with a real human body. Besides, even if the avatar could be confused with a real body, it would not look like the user. Thus, its contribution to kinaesthesia (and its embodiment) attests that some semantic incongruencies do not hinder multisensory integration. It should be noted that in Giroux et al. (2018) kinaesthetic illusions were comparable to those observed in the traditional mirror paradigm (Guerraz et al., 2012; Metral et al., 2015), even though they were slightly less frequent ( $85 \%$ of trials compared to $96 \%$ in Guerraz et al., 2012 and Metral et al., 2015) and slower (mean estimated speed of 6.3 compared to 7 in Guerraz et al., 2012 or 8.1 in Metral et al., 2015). In the traditional mirror paradigm, a mirror is placed in front of the participants, in its sagittal axis, in such a way that they can see the reflection of their left arm while their right arm is hidden behind the mirror. In this configuration, the reflection of the left arm appears to be the hidden right arm and when the left arm is passively moved, its reflection moves too and an illusion of movement can 
appear in the right, static arm of the participants. The visual motion cues coming from the mirror are integrated with proprioceptive ones coming from the contralateral arm, like in our virtual mirror paradigm. In the mirror paradigm, the visual information comes from a realistic and very similar arm since it is the left arm reflection. Using an avatar, appears to generate comparable illusions of movement, the visual motion cues coming from the avatar being integrated with the contralateral proprioceptive motion cues like in the traditional mirror paradigm. Moreover, the avatar does not need to be morphologically similar to a human body since illusions still occur when its forearms are only represented by a point light display (Giroux et al., 2019).

Secondly, when it comes to spatial congruency, the results presented earlier indicate that visual motion cues do not need to come from the same location in space to be integrated for kinaesthesia. Indeed, proprioceptive signals are coming from the real body of the participant and, visual motion cues coming from the first-person perspective avatar used in Giroux et al $(2018,2019)$ are, by definition, collocated with the real body. In our most recent studies (Giroux et al. 2021), we showed that visual motion cues are integrated with the proprioceptive signals, even when they are only seen through a mirror facing the participant and no more directly from a first-person perspective avatar. This result is not very surprising when we think about our daily use of mirror for combing our hair, putting on makeup or shaving. Truly, the visual information conveyed by a mirror refers to our own body location in space. In this respect, the spatial incongruency may be limited in this condition, accounting for the multisensory integration between visual and proprioceptive signals. Nevertheless, the second experiment showed that visual motion cues coming from a third-person perspective avatar were also used for kinaesthesia even though they do not refer to the space of the body anymore. Quite important spatial incongruencies do not abolish the multisensory integration for kinaesthesia in this case. However, we have to note that when the third-person perspective avatar is seen in profile, on one side of the participant, the contribution of visual motion cues appears far less obvious. In addition, a first-person perspective avatar generates more intense illusions than when it is seen through a mirror or at the third-person perspective and it seems that the less spatial congruency there is, the less intense the illusions are. Although these studies cannot prove the existence of a continuum, they suggest that there are different degrees of congruency, either semantic or spatial, that have a different impact on kinaesthesia. In a probabilistic view of perception, in which the weight allocated to a sensory modality in multisensory integration depends on its reliability (Ernst et Banks, 2002), our results can be explained by a lower weight allocated to semantically or spatially incongruent visual information.

In conclusion, all these results taken together indicate that any relevant signal which refers in some way or another to the body, as by the embodiment phenomenon, can be used to perceive one's own movements. Nevertheless, the impact of such signal on the movement perception depends on its congruency with other bodily motion signals or similarity to natural and usual ones.

\section{References}

Barra Julien, Giroux Marion, Metral Morgane, Cian Corinne, Luyat Marion, Kavounoudias Anne, Guerraz Michel. (2020). Functional properties of extended body representations in the context of kinesthesia. Neurophysiologie Clinique / Clinical Neurophysiology, vol. 50, n6, p. 455-465. https://doi.org/10.1016/j.neucli.2020.10.011

Beaudoin Marine, Barra Julien, Dupraz Louise, Mollier-Sabet Pauline, Guerraz Michel. (2020). The impact of embodying an "elderly" body avatar on motor imagery. Experimental Brain Research, vol. 238, n6, p. 14671478. https://doi.org/10.1007/s00221-020-05828-5

Bevilacqua Frédéric, Segalen Maël, Marchand-Pauvert Véronique, Peyre Iseline, Pradat-Diehl Pascale, Roby-Brami Agnès. (2018). Exploring Different Movement Sonification Strategies for Rehabilitation in Clinical Settings. Proceedings of the 5th International Conference on Movement and Computing, $\mathrm{n}^{\circ}$ 42, $\mathrm{p} .1-6$. https://doi.org/10.1145/3212721.3212881

Blanchard Caroline, Roll Régine, Roll Jean-Pierre, Kavounoudias Anne. (2013). Differential Contributions of Vision, Touch and Muscle Proprioception to the Coding of Hand Movements. PLoS ONE, vol. 8, n²4, p. e62475. https://doi.org/10.1371/journal.pone.0062475

Bock Otmar, Pipereit Katja, Mierau Andreas. (2007). A method to reversibly degrade proprioceptive feedback in research on human motor control. Journal of Neuroscience Methods, vol. 160, n², p. 246-250. https://doi.org/10.1016/j.jneumeth.2006.09.010

Botvinick Matthew, Cohen Jonathan. (1998). Rubber hands 'feel' touch that eyes see. Nature, vol. 391, n 6669, p. 756. https://doi.org/10.1038/35784

Brandt T, Dichgans J, Koenig E. (1972). Perception of self-rotation (circular vection) induced by optokinetic stimuli. Pflugers Archiv : European Journal of Physiology, vol. 332, p. Suppl 332:R98-Suppl 332:R98.

Brun Clémentine, Guerraz Michel. (2015). Anchoring the "floating arm": Use of proprioceptive and mirror visual feedback from one arm to control involuntary displacement of the other arm. Neuroscience, vol. 310, p. 268278. https://doi.org/10.1016/j.neuroscience.2015.09.052

Brun Clémentine, Metral Morgane, Chancel Marie, Kavounoudias Anne, Luyat Marion, Guerraz Michel. (2015). Passive or simulated displacement of one arm (but not its mirror reflection) modulates the involuntary motor 
behavior of the other arm. Neuroscience, vol. 285, p. 343-355. https://doi.org/10.1016/j.neuroscience.2014.11.036

Calvin-Figuière Sarah, Romaiguère Patricia, Gilhodes Jean-Claude, Roll Jean-Pierre. (1999). Antagonist motor responses correlate with kinesthetic illusions induced by tendon vibration. Experimental Brain Research, vol. 124, n³, p. 342-350. https://doi.org/10.1007/s002210050631

Carruthers Glenn. (2009). Is the body schema sufficient for the sense of embodiment? An alternative to de Vignemont's model. Philosophical Psychology, vol. 22, n² 2, p. 123-142. https://doi.org/10.1080/09515080902802785

Chancel Marie, Blanchard Caroline, Guerraz Michel, Montagnini Anna, Kavounoudias Anne. (2016). Optimal visuotactile integration for velocity discrimination of self-hand movements. Journal of Neurophysiology, vol. 116, n³, p. 1522-1535. https://doi.org/10.1152/jn.00883.2015

Collins, Davide. F, Prochazka Arthur. (1996). Movement illusions evoked by ensemble cutaneous input from the dorsum of the human hand. The Journal of Physiology, vol. 496, $\mathrm{n}^{\circ}$ 3, p. 857-871. https://doi.org/10.1113/jphysiol.1996.sp021733

de Vignemont Frédérique. (2010). Body schema and body image_Pros and cons. Neuropsychologia, vol. 48, $\mathrm{n}^{\circ} 3, \mathrm{p}$. 669-680. https://doi.org/10.1016/j.neuropsychologia.2009.09.022

de Vignemont Frédérique. (2011). Embodiment, ownership and disownership. Consciousness and Cognition, vol. 20, $\mathrm{n}^{\circ}$ 1, p. 82-93. https://doi.org/10.1016/j.concog.2010.09.004

Effenberg, Alfred. O. (2004). Using Sonification to Enhance Perception and Reproduction Accuracy of Human Movement Patterns. 5.

Effenberg Alfred. O., Schmitz Gerd, Baumann Florian, Rosenhahn Bodo, Kroeger Daniela. (2015). SoundScriptSupporting the acquisition of character writing by multisensory integration. Open Psychology Journal 8 (2015), Nr. 1, vol. 8, $\mathrm{n}^{\circ}$ 1, p. 230-237. http://dx.doi.org/10.15488/817

Ernst Marc O., Banks Martin S. (2002). Humans integrate visual and haptic information in a statistically optimal fashion. Nature, vol. 415 , p. 429.

Farmer Harry, Tajadura-Jiménez Ana, Tsakiris Manos. (2012). Beyond the colour of my skin: How skin colour affects the sense of body-ownership. Consciousness and Cognition, vol. 21, n ${ }^{\circ}$ 3, p. 1242-1256. https://doi.org/10.1016/j.concog.2012.04.011

Gallagher Maria, Dowsett Ross, Ferrè Elisa Raffaella. (2019). Vection in virtual reality modulates vestibular-evoked myogenic potentials. European Journal of Neuroscience, vol. 50, n ${ }^{\circ}$ 10, p. 3557-3565. https://doi.org/10.1111/ejn.14499

Gallagher Shaun. (2005). How the Body Shapes the Mind. In How the Body Shapes the Mind. Oxford University Press. doi:10.1093/0199271941.001.0001

Giroux Marion, Barra Julien, Barraud Pierre-Alain, Graff Christian, Guerraz Michel. (2019). From Embodiment of a Point-Light Display in Virtual Reality to Perception of One's Own Movements. Neuroscience, vol. 416, p. 3040. https://doi.org/10.1016/j.neuroscience.2019.07.043

Giroux Marion, Barra Julien, Graff Christian, Guerraz Michel. (2021). Multisensory integration of visual cues from first- to third-person perspective avatars in the perception of self-motion. Attention, Perception, \& Psychophysics, vol. 83, p. 2634-2655. https://doi.org/10.3758/s13414-021-02276-3

Giroux Marion, Barra Julien, Zrelli Issam-Eddine, Barraud, Pierre-Alain, Cian Corinne, Guerraz Michel. (2018). The respective contributions of visual and proprioceptive afferents to the mirror illusion in virtual reality. PLOS ONE, vol. 13, n 8, p. e0203086. https://doi.org/10.1371/journal.pone.0203086

Gonzalez-Franco Mar, Peck Tabitha C. (2018). Avatar Embodiment. Towards a Standardized Questionnaire. Frontiers in Robotics and AI, 5. https://doi.org/10.3389/frobt.2018.00074

Guerraz Michel, Provost S, Narison Rindra, Brugnon A, Virolle S, Bresciani Jean-Pierre. (2012). Integration of visual and proprioceptive afferents in kinesthesia. Neuroscience, vol. 223, p. 258-268. https://doi.org/10.1016/j.neuroscience.2012.07.059

Guerraz Michel, Bronstein Adolfo M. (2008). Mechanisms underlying visually induced body sway. Neuroscience Letters, vol. 443, $\mathrm{n}^{\circ}$ 1, p. 12-16. https://doi.org/10.1016/j.neulet.2008.07.053

Harquel Sylvain, Guerraz Michel, Barraud Pierre-Alain, Cian Corinne. (2020). Modulation of alpha waves in sensorimotor cortical networks during self-motion perception evoked by different visual-vestibular conflicts. Journal of Neurophysiology, vol. 123, $\mathrm{n}^{\circ}$ 1, p. 346-355. https://doi.org/10.1152/jn.00237.2019

Head Henry, Holmes Gordon. (1911). Sensory Disturbances From Cerebral Lesions. Brain, vol. 34, n 2-3, p. $102-254$. https://doi.org/10.1093/brain/34.2-3.102

Izumizaki Masahiko, Tsuge Mikio, Akai Lena, Proske Uwe, Homma Ikuo. (2010). The illusion of changed position and movement from vibrating one arm is altered by vision or movement of the other arm: Plasticity of vibration illusion. The Journal of Physiology, vol. 588, n 15, p. 2789-2800. https://doi.org/10.1113/jphysiol.2010.192336 
Kavounoudias Anne, Roll Jean-Pierre, Anton J. L, Nazarian B, Roth M, Régine Roll. (2008). Proprio-tactile integration for kinesthetic perception: An fMRI study. Neuropsychologia, vol. 46, $\mathrm{n}^{\circ} \quad 2, \quad \mathrm{p} . \quad 567-575$. https://doi.org/10.1016/j.neuropsychologia.2007.10.002

Kilteni Konstantina, Groten Raphaela, Slater Mel. (2012). The Sense of Embodiment in Virtual Reality. Presence: Teleoperators and Virtual Environments, vol. 21, n 4, p. 373-387. https://doi.org/10.1162/PRES_a_00124

Kilteni Konstantina, Normand Jean-Marie, Sanchez-Vives Maria V., Slater Mel. (2012). Extending Body Space in Immersive Virtual Reality: A Very Long Arm Illusion. PLOS ONE, vol. 7, $\mathrm{n}^{\circ}$ 7, p. e40867. https://doi.org/10.1371/journal.pone.0040867

Kuiper Ouren X., Bos Jelte E., Diels Cyriel. (2019). Vection does not necessitate visually induced motion sickness. Displays, vol. 58, p. 82-87. https://doi.org/10.1016/j.displa.2018.10.001

Lackner James R., DiZio Paul. (1984). Some efferent and somatosensory influences on body orientation and oculomotor control. Sensory Experience, Adaptation and Perception., p. 281-301.

Lin Lorraine, Jörg Sophie. (2016). Need a Hand?: How Appearance Affects the Virtual Hand Illusion. Proceedings of the ACM Symposium on Applied Perception, p. 69-76. https://doi.org/10.1145/2931002.2931006

Lira Marilia, Egito Julia H, Dall’Agnol Patricia A, Amodio David M, Gonçalves Óscar F, Boggio Paulo S. (2017). The influence of skin colour on the experience of ownership in the rubber hand illusion. Scientific Reports, vol. 7, ${ }^{\circ}$ 1, p. 15745. https://doi.org/10.1038/s41598-017-16137-3

Lloyd Donna M. (2007). Spatial limits on referred touch to an alien limb may reflect boundaries of visuo-tactile peripersonal space surrounding the hand. Brain and Cognition, vol. 64; $\mathrm{n}^{\circ}$ 1, p. 104-109. https://doi.org/10.1016/j.bandc.2006.09.013

Longo Matthew R, Schüür Friederike, Kammers Marjolein P. M, Tsakiris Manos, Haggard Patrick. (2008). What is embodiment? A psychometric approach. Cognition, vol. 107, n 3, p. 978-998. https://doi.org/10.1016/j.cognition.2007.12.004

Lopez Christophe, Schreyer Helene-Marianne, Preuss Nora, Mast Fred W. (2012). Vestibular stimulation modifies the body schema. Neuropsychologia, vol. $\quad 50, \quad \mathrm{n}^{\circ} \quad 8, \quad$ p. $\quad 1830-1837$. https://doi.org/10.1016/j.neuropsychologia.2012.04.008

Lugrin Jean-Luc, Latt Johanna, Latoschik Marc Erich. (2015). Avatar anthropomorphism and illusion of body ownership in VR. 2015 IEEE Virtual Reality (VR), p. 229-230. https://doi.org/10.1109/VR.2015.7223379

Ma Ke, Hommel Bernhard. (2015). The role of agency for perceived ownership in the virtual hand illusion. Consciousness and Cognition, vol. 36, p. 277-288. https://doi.org/10.1016/j.concog.2015.07.008

Maselli Antonella, Slater Mel. (2013). The building blocks of the full body ownership illusion. Frontiers in Human Neuroscience, 7. https://doi.org/10.3389/fnhum.2013.00083

Maulucci Ruth A, Eckhouse Richard H. (2001). Retraining reaching in chronic stroke with real-time auditory feedback. NeuroRehabilitation, vol. 16, $\mathrm{n}^{\circ} 3$, p. 171-182.

Metral Morgane, Chancel Marie, Brun Clémentine, Luyat Marion, Kavounoudias Anne, Guerraz Michel. (2015). Kinaesthetic mirror illusion and spatial congruence. Experimental Brain Research, vol. 233, n 5, p. $1463-1470$. https://doi.org/10.1007/s00221-015-4220-1

Metral Morgane, Guerraz Michel (2019). Fake hand in movement: Visual motion cues from the rubber hand are processed for kinesthesia. Consciousness and Cognition, vol. 73, p. 102761. https://doi.org/10.1016/j.concog.2019.05.009

Normand Jean-Marie, Giannopoulos Elias, Spanlang Bernhard, Slater Mel. (2011). Multisensory Stimulation Can Induce an Illusion of Larger Belly Size in Immersive Virtual Reality. PLOS ONE, vol. 6, n 1, p. e16128. https://doi.org/10.1371/journal.pone.0016128

Peck Tabitha C, Seinfeld Sofia, Aglioti Salvatore M, and Slater Mel. (2013). Putting yourself in the skin of a black avatar reduces implicit racial bias. Consciousness and Cognition, vol. 22, n ${ }^{\circ} 3$, p. $779-787$. https://doi.org/10.1016/j.concog.2013.04.016

Preston Catherine. (2013). The role of distance from the body and distance from the real hand in ownership and disownership during the rubber hand illusion. Acta Psychologica, vol. 142, $\mathrm{n}^{\circ} \quad 2, \mathrm{p} .177-183$. https://doi.org/10.1016/j.actpsy.2012.12.005

Proske Uwe, Gandevia Simon C. (2012). The Proprioceptive Senses: Their Roles in Signaling Body Shape, Body Position and Movement, and Muscle Force. Physiological Reviews, vol. 92, n 4, p. 1651-1697. https://doi.org/10.1152/physrev.00048.2011

Röder Brigitte, Pagel Birthe, Heed Tobias (2013). The implicit use of spatial information develops later for crossmodal than for intramodal temporal processing. Cognition, vol. 126, $\mathrm{n}^{\circ} 2$, p. 301-306. https://doi.org/10.1016/j.cognition.2012.09.009

Roll Régine, Kavounoudias Anne, Roll Jean-Pierre. (2002). Cutaneous afferents from human plantar sole contribute to body posture awareness. NeuroReport, vol. 13, n 15, p. 1957. 
Schwind Valentin, Lin Lorraine, Di Luca Massimiliano, Jörg Sophie, Hillis James. (2018). Touch with Foreign Hands: The Effect of Virtual Hand Appearance on Visual-haptic Integration. Proceedings of the 15th ACM Symposium on Applied Perception, p. 9:1-9:8. https://doi.org/10.1145/3225153.3225158

Sforza Anna, Bufalari Ilaria, Haggard Patrick, Aglioti Salvatore M. (2010). My face in yours: Visuo-tactile facial stimulation influences sense of identity. Social Neuroscience, vol. 5, n ${ }^{\circ} \quad 2, \quad$ p. $148-162$. https://doi.org/10.1080/17470910903205503

Shimada Sotaro, Suzuki Tatsuya, Yoda Naohiko, Hayashi Tomoya. (2014). Relationship between sensitivity to visuotactile temporal discrepancy and the rubber hand illusion. Neuroscience Research, vol. 85, p. 33-38. https://doi.org/10.1016/j.neures.2014.04.009

Slater Mel, Spanlang Bernhard, Sanchez-Vives Maria V, Blanke Olaf. (2010). First Person Experience of Body Transfer in Virtual Reality. PLOS ONE, vol. 5, n 5, p. e10564. https://doi.org/10.1371/journal.pone.0010564

Tajadura-Jiménez Ana, Tsakiris Manos, Marquardt Torsten, Bianchi-Berthouze Nadia. (2015). Action sounds update the mental representation of arm dimension: Contributions of kinaesthesia and agency. Frontiers in Psychology, 6. https://doi.org/10.3389/fpsyg.2015.00689

Tardy-Gervet Marie Françoise, Gilhodes Jean Claude, Roll Jean-Pierre. (1984). Perceptual and motor effects elicited by a moving visual stimulus below the forearm: An example of segmentary vection. Behavioural Brain Research, vol. 11, n 2, p. 171-184. https://doi.org/10.1016/0166-4328(84)90139-6

Tsakiris Manos. (2008). Looking for Myself: Current Multisensory Input Alters Self-Face Recognition. PLOS ONE, vol. 3, n 12, p. e4040. https://doi.org/10.1371/journal.pone.0004040

Tsakiris Manos, Carpenter Lewis, James Dafydd, Fotopoulou Aikaterini. (2010). Hands only illusion: Multisensory integration elicits sense of ownership for body parts but not for non-corporeal objects. Experimental Brain Research, vol. 204, n 3, p. 343-352. https://doi.org/10.1007/s00221-009-2039-3

Väljamäe Aleksander. (2009). Auditorily-induced illusory self-motion: A review. Brain Research Reviews, vol. 61, $\mathrm{n}^{\circ}$ 2, p. 240-255. https://doi.org/10.1016/j.brainresrev.2009.07.001

Yee Nick, Bailenson Jeremy. (2007). The Proteus Effect: The Effect of Transformed Self-Representation on Behavior. Human Communication Research, vol; 33, $\mathrm{n}^{\circ} 3$, p. 271-290. https://doi.org/10.1111/j.1468-2958.2007.00299.x

Zhang Jing, Hommel Bernhard. (2016). Body ownership and response to threat. Psychological Research, vol. 80, ${ }^{\circ} 6$, p. 1020-1029. https://doi.org/10.1007/s00426-015-0698-1 